\title{
MODELLING OF HYDROCARBON GAS AND LIQUID LEAKS FROM PRESSURIZED PROCESS SYSTEMS
}

\author{
ANAND BAHUGUNI, AMARANATH S. KUMARA \& KNUT E. T. GILJARHUS \\ Lloyd's Register Consulting, Norway. \\ Lloyd's Register Global Technology Centre, Singapore. \\ University of Stavanger, Norway.
}

\begin{abstract}
The hydrocarbon leaks from process systems potentially lead to hazardous consequences with regard to human safety, environmental pollution and valuable assets. The hydrocarbon leaks may be gas leaks, liquid leaks or multiphase leaks. The gas leaks have the highest potential of damage due to explosion accidents. Both gas and oil leaks can create long-lasting fires threatening personnel safety and structural integrity of process plants and offshore platforms. One common method for limiting the consequences associated with a process emergency is the rapid depressurization or blowdown of pressurized process systems. There is experimental evidence that the assumption of thermodynamic equilibrium is not appropriate during rapid depressurization, since the two phases show an independent temperature evolution. The current work proposes a model for the simulation of the blowdown of vessels containing two-phase (gas-liquid) hydrocarbon fluids, considering partial phase equilibrium between phases. Two phases may be present either already at the beginning of the blowdown process (for instance in gasliquid separators) or as the liquid is formed from flashing of the vapour due to the cooling induced by pressure decrease. In addition, the transient behaviour of hydrocarbon leaks from pressurized process systems during depressurization is also included in the model providing the inputs for risk assessments. The model is based on a compositional approach, and it takes into account coupled effects of internal heat and mass transfer processes, as well as heat transfer with the vessel wall and the external environment. The vapour liquid equilibria calculations are performed using dynamic link library provided by the comprehensive pressure volume temperature and physical properties package 'Multiflash'. Numerical simulations show a generally good agreement with experimental measurements.

Keywords: hydrocarbon leak, heat transfer, partial phase equilibrium, thermodynamics.
\end{abstract}

\section{INTRODUCTION}

Hydrocarbon processing facilities pose severe risks with respect to fire, explosions and vessel ruptures. Among the prime methods to prevent and limit the loss potential from such incidents are the provisions of hydrocarbon inventory isolation and removal systems. These systems are commonly referred to in the petroleum industry as emergency shutdown and depressurization or blowdown. The term 'blowdown' refers to the emergency or planned depressurization of process equipment, such as vessels, heat exchangers, distillation columns, and compressors, in order to remove combustible hydrocarbons and protect against excessively high pressures or temperatures. This may be necessary, for example, in the event of a fire, leak, pipe rupture, or other hazardous situations, as well as for a planned shutdown. Although the blowdown process is intended to ensure the safe operation of the plant, it is itself a potentially hazardous operation, during which a number of concerns arise. During depressurization, the temperature of the fluid can drop significantly. The heat transfer between the fluid and the vessel wall leads to a reduction in the temperature of the wall, which can lead to a fracture if the wall temperature falls below the ductile-brittle transition temperature of steel. It is therefore critical to accurately determine the lowest temperatures that can be reached during blowdown so that the proper construction materials can be selected [1]. 
The risk assessment of an accidental scenario starts with modelling of transient leak rates. This may be hydrocarbon releases from different sources such as pipes and associated fittings, vessels and pipeline/risers. The phase of the leaking medium is the next important aspect. The hydrocarbon leaks may be gas leaks, liquid leaks or multiphase leaks. Different models suitable for the different phase compositions and the different sources (mainly reflecting the difference between outflow from a vessel or from a pipeline or pipe section) have to be used. The models are primarily aimed at determining the flow rate as a function of time. There are several factors which influence the flow modelling and influence the duration of the leak. The isolation and depressurization will limit the volume of gas and oil escaping from the leak. The depressurization model is the most difficult part in both simple and complex hydrocarbon leak calculation tools [2]. The realistic modelling of the leak and its duration is obviously very important to determine the size and duration of fire, explosion overpressure and the response of the load bearing structures of the platforms and process plants.

Modelling a system during depressurization can be quite complex. During blowdown, a multiphase mixture of gas, liquid and aqueous phases flow through the vessel, valve and any associated piping. As the fluid flows, heat transfer between the fluid and vessel wall, as well as between the different fluid phases, will occur. As liquid evaporates and vapour condenses, an accompanying mass transfer between the phases will also occur. Both the fluid mechanics and heat and mass transfer effects are time-dependent and three-dimensional. Additional modelling complexity is introduced for high pressure systems, where the thermodynamic trajectory during the depressurization takes the fluid near or through the critical region [1]. In recent years a number of theoretical and experimental studies have addressed the depressurization issues. The theoretical models primarily fall into two categories. The first is based on the simplified relations which are either incapable of predicting wall and fluid temperatures accurately or are applicable to non-condensable gases only $[3,4]$. The second category is the one based on rigorous analytical procedures such as those proposed by Haque et al. [5], Overa et al. [6], Speranza et al. [7], Wong [8] and Berge et al. [12]. The mathematical model, BLOWDOWN, developed by Haque et al. [5] is aimed at providing accurate predictions of physically significant effects taking place during blowdown. The authors claim that their model allows for a multicomponent system, non-equilibrium conditions between the gas and the liquid within the vessel, and possibility of having separate water as well as gas and liquid phase. Also all the heat transfer mechanisms, except for the one between the two fluids, are implemented in the model. The numerical results obtained by the authors show a good agreement with some experiments [5]. Speranza et al. [7] proposed a model for the simulation of the blowdown of vessels containing two-phase hydrocarbon fluids, considering non equilibrium between phases. The model is partially based on the description of the model by Haque et al. [5], and it takes into account internal heat and mass transfer processes, as well as heat transfer with the vessel wall and the external environment. The model has been validated against experimental measurements. Heat exposure of process equipment during depressurization is a complex problem area that has gained interest over the last years. VessFire has been developed for time-dependent, non-linear analysis of thermo-mechanical response of process segments and process equipment (vessels and piping) during blowdown, with or without exposure to fire [12].

The current work proposes a model for the simulation of the blowdown of vessels containing two-phase (gas-liquid) hydrocarbon fluids, considering partial phase equilibrium (PPE) between phases. Two phases may be present either already at the beginning of the blowdown process (for instance in gas-liquid separators) or as the liquid is formed from flashing of the vapour due to the cooling induced by pressure decrease. In addition, the transient behaviour 
of hydrocarbon leaks from pressurized process systems during depressurization is also included in the model providing the inputs required for risk assessments. The model is based on a compositional approach, and it takes into account coupled effects of internal heat and mass transfer processes, as well as heat transfer with the vessel wall and the external environment. The vapour liquid equilibria (VLE) calculations are performed using dynamic link library (DLL) provided by the comprehensive pressure volume temperature (PVT) and physical properties package 'Multiflash'. Numerical predictions are validated against the experimental measurements.

\section{MATHEMATICAL MODEL}

The hydrocarbon mixture inside the pressure vessel is a complex mixture of various components. At equilibrium, the gas and the liquid phases coexist inside the container. The heat transfer into the liquid or the pressure drop causes the liquid to evaporate. Similarly, the vapour surrounding the liquid condenses due to heat transfer or pressure increase.

When the blow down or leak occurs there is a sudden loss of pressure in the container. Also, the loss of internal energy due to the lost mass/energy of hydrocarbon leads to sudden drop of vessel temperature. This drop in temperature and pressure leads to vapour condensation in the vessel. Hence to simulate the hydrocarbon leak process, accurate prediction of the phases and the composition of each phase are very important. The heat transfer process includes the heat exchange between the wall of the container and the liquid, the wall of the container and vapour and between the liquid and the vapour at the interphase. Figure 1 shows an illustration of the physical processes occurring during a hydrocarbon leak from a pressurized process vessel.

\subsection{Partial phase equilibrium}

Various existing methods for the prediction of the state of the mixture assume the mixture to be homogeneous, that is, having a single temperature across the phases. In reality, gas being

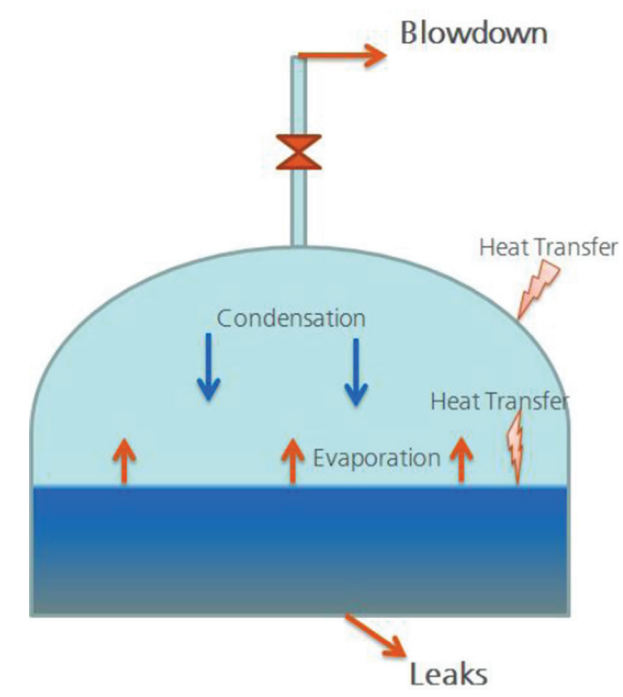

Figure 1: Schematic of the heat transfer process in a pressure vessel during depressurization. 
a low specific heat fluid will either cool down or heat up much faster than the liquid. Also, the heat transfer rates for the gas and the liquid are different. These inequalities lead to significant temperature differences for the gas and liquid phases (and hence the internal energy and density). Hence, when complete equilibrium calculations are used in leak simulations, the compositions and the leak rates will be inaccurate.

In the PPE approach, the gas and the liquid phases are treated separately. But, as shown in Fig. 1, the condensation of the gas and the evaporation of the liquid inside the container occur simultaneously. The condensed gas and the evaporated liquid need to be mixed with their respective phases when the PPE approach is used. The entire process will be quasi-static. The gas and the liquid phases are treated separately for the heat transfer, blowdown and leak calculations. The temperature of the gas and the liquid are also treated separately. This leads to accurate heat transfer prediction between the gas to the container wall, liquid to the container wall and gas to liquid. Also, since the compositions are treated separately for the two phases, the leak compositions and mass flow rates are predicted accurately. The two bulk phases, gas and liquid, produce the vapour condensate and the evaporated liquid as the daughter phases. The two daughter phases are named as 'condensate' and the 'vapour'. The daughter phases appear only instantaneously, i.e. they merge with respective parent phases quickly. The 'condensate' which is a daughter phase from the gas, immediately merges with the liquid phase and similarly the 'vapour' phase which is a daughter phase of liquid merges with the gas. When the daughter phases merge with the parent phases, the temperature they acquire is assumed to be that of the parent phase with which they merge. The compositions, energies and volumes of the parent phases are thus adjusted every time-step taking into account the merged daughter phases.

The PPE simulations involve the flash calculations to determine the state of the mixture at every time step. The Peng Robinson or the Soave Redlich Kwong models are used as the equations of state for the hydrocarbon mixtures. These models are widely used in the oil and gas industry for modelling the hydrocarbon behaviour. Implementing the flash calculations involve libraries with hydrocarbon properties, binary interaction parameters and iterative solution procedures with stable convergence. Hence, VLE calculations are performed using DLL provided by the comprehensive PVT and physical properties package 'Multiflash'. Multiflash being accurate, fast, comprising of comprehensive database of hydrocarbons and equations of state improves the computational efficiency and accuracy of the model.

\subsection{Mathematical model}

The mathematical model is based on the quasi-static equilibrium assumptions or PPE. The conservation relations of mass and energy are detailed in this section. For PPE, the temperature for the gas phase and the liquid phase are treated separately. The pressure is calculated as a single entity. The conservation equations for the mixture are as below:

$$
\begin{aligned}
& \frac{\mathrm{d} n_{1}}{d t}=-Q_{1}, \\
& \frac{\mathrm{d} n_{\mathrm{g}}}{d t}=-Q_{\mathrm{g}}, \\
& \frac{\mathrm{d} U_{\mathrm{g}}}{d t}=q_{\mathrm{g}}-U_{\text {gLeak }},
\end{aligned}
$$




$$
\frac{\mathrm{d} U_{1}}{d t}=q_{1}-U_{\text {ILeak }}
$$

where $n_{1}$ and $n_{\mathrm{g}}$ are the number of moles of liquid and gas inside the container, $Q_{1}$ and $Q_{\mathrm{g}}$ are the molar flow rates of liquid and gas due to leak or blowdown, respectively, $U_{1}$ and $U_{\mathrm{g}}$ are the internal energies of the liquid and the gas, $U_{\text {gLeak }}$ and $U_{\text {ILeak }}$ are the energy lost due to gas and liquid leaks, respectively, $g_{\mathrm{g}}$ and $q_{1}$ are the heat added to the gas and liquid phases due temperature gradients, respectively. The heat transfer to the gas and liquid phases are made of two components, one is the heat transfer with the wall of the container $\left(g_{\mathrm{gw}}\right.$ and $\left.q_{\mathrm{lw}}\right)$ and the other is the heat transfer between the gas and the liquid phases $\left(g_{\mathrm{lg}}\right)$. Thus, $q_{\mathrm{g}}=q_{\mathrm{gw}}+q_{\mathrm{gl}}$ and $q_{1}=q_{\mathrm{lw}}+q_{\mathrm{lg}}$. The spatial variation of either the temperature or the compositions in the mixture are not accounted in the current work, i.e. the temperature and the compositions vary only for different phases and for a given phase they are uniform everywhere. Only the conduction and the convection heat transfer modes are included in the model. The radiation heat transfer is not accounted for in the model as it has negligible effect unless the fire hazards are considered, i.e. if a fire directly impacts the process equipment under consideration.

\subsubsection{Gas blowdown/leak rate modelling}

The gas leaks and the blowdown valve are modelled as converging flow passages with a given opening diameter and the discharge coefficient $\left(C_{\mathrm{d}}\right)$. The gas flow can be identified as either critical (choked) or subcritical (non-choked) conditions. The two conditions are identified based on the ratio of ambient pressure to the tank pressure. The gas flow rates are modelled as below.

For a subcritical flow, if

$$
\begin{gathered}
\frac{\mathrm{P}_{\mathrm{atm}}}{P}>\left(\frac{2}{\gamma+1}\right)^{\frac{\gamma}{\gamma-1}}, \\
\mathrm{Q}_{\mathrm{g}}=C_{\mathrm{dg}} A \sqrt{\rho p Z} \sqrt{\frac{\gamma}{\gamma+1}} \sqrt{\left(\frac{p_{\mathrm{atm}}}{p}\right)^{\frac{2}{\gamma}}-\left(\frac{p_{a t m}}{p}\right)^{\frac{\gamma+1}{\gamma}}} .
\end{gathered}
$$

Otherwise, the flow is choked or supercritical.

$$
\mathrm{Q}_{\mathrm{g}}=C_{\mathrm{dg}} A \sqrt{\gamma \rho p Z}\left(\frac{2}{\gamma+1}\right)^{\frac{\gamma+1}{2(\gamma-1)}}
$$

where $p$ and $p_{\text {atm }}$ are the container pressure and the atmospheric pressure, $\gamma$ is the ratio of specific heat capacities $\left(C_{\mathrm{p}} / C_{\mathrm{v}}\right), C_{\mathrm{dg}}$ is the coefficient discharge for gas leaks $\left(C_{\mathrm{dg}}=0.81\right)$, $\rho$ is the density of the gas, $A$ is the cross-sectional area and $Z$ is the compressibility factor. The properties such as density, specific heat capacity ratio and the compressibility factor are calculated with Multiflash software as a function of container pressure $(p)$ and temperature. The cross-sectional area is the simplified circular area for a defined leak diameter.

\subsubsection{Liquid leak rate modelling}

The liquid flow rate from the leak is a function of the container pressure, temperature and the liquid height. The liquid height $(h)$ is determined from the volume fraction and the geometry 
of the tank. The tank geometry in the current study assumes flat ends on two sides. If the container is oriented vertically, then the liquid height from the volume is calculated with simple formula as

$$
h=\frac{4 V}{\pi D^{2}},
$$

where $V$ is the liquid volume and $D$ is the container diameter. If the container is horizontally oriented, then the height calculation will involve finding the root for

$$
V-\left[r^{2} \theta-(r-h) \sqrt{2 r h-h^{2}}\right] l=0,
$$

where

$$
\theta=\cos ^{-1}\left(\frac{r-h}{r}\right)
$$

Figure 2 demonstrates the liquid height calculation for the horizontal and vertical containers. The liquid leak rate is then given by eqn (11). The default discharge coefficient value $\left(C_{\mathrm{dl}}\right)$ used for liquid flow calculation is 0.61 .

$$
Q_{1}=C_{\mathrm{dl}} A \rho \sqrt{2\left(\frac{p-p_{\mathrm{atm}}}{\rho}+g h\right)} .
$$

\subsubsection{Heat transfer modelling}

The gas and the liquid phases of the mixture interact differently with the surrounding based on their heat transfer capacities. The different phases exchange heat with the surrounding through the wall of the container and also among themselves through the phase interface. The heat transfer modes during depressurization are illustrated in Fig. 1. The heat transfer modes considered in this analysis are mainly the conduction and the convection. The radiation heat transfer being very insignificant contributor has been neglected. If the fire hazards are to be simulated, then the radiation heat transfer would be a dominant mode of heat exchange. The heat capacity of the liquid phase is higher than that of the gas phase. Hence, the heating or cooling effects alter the gas temperature faster than that of the liquid (for the same area of heat transfer). This in turn affects the internal energy and the density of the gas. The leak rate
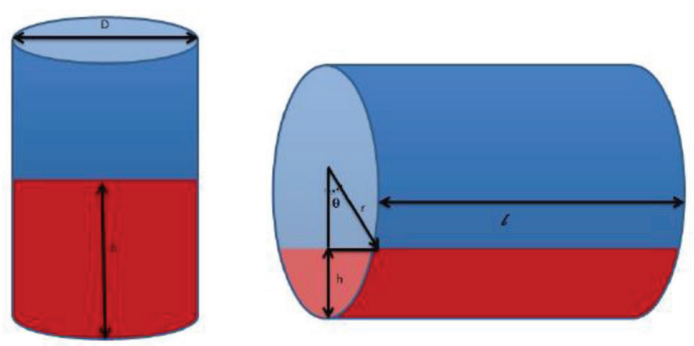

Figure 2: Liquid height calculations for vertical and horizontal containers. 
is a function of pressure and gas density. The pressure is calculated from the internal energy and the specific volume of the gas. Hence, accurate calculation of gas temperature (along with composition) is essential in determining the container pressure and leak rates at any given time. The heat transfer between the gas, liquid and the container wall is given by

$$
\begin{gathered}
q_{\mathrm{gw}}=h_{\mathrm{gw}} A_{\mathrm{gw}}\left(T_{\mathrm{gw}}-T_{\mathrm{g}}\right), \\
q_{\mathrm{lw}}=h_{\mathrm{lw}} A_{\mathrm{lw}}\left(T_{\mathrm{lw}}-T_{1}\right),
\end{gathered}
$$

where $q_{g w}$ and $q_{l w}$ are the heat transfer rates for gas and liquid phases with the container wall, $A_{\mathrm{gw}}$ and $A_{\mathrm{lw}}$ are the corresponding heat transfer areas, $h_{g w}$ and $h_{l w}$ are the heat transfer coefficients, respectively, and $T_{g w}$ and $T_{l w}$ are the film temperatures at the gas-wall and liquid-wall interfaces, respectively. At the gas-liquid interface, the smaller heat transfer coefficient among the gas and liquid phases is used. The heat transfer rate at the gas-liquid interface is thus

$$
q_{\mathrm{lg}}=h_{\mathrm{lg}} A_{\mathrm{lg}}\left(T_{\mathrm{g}}-T_{1}\right)
$$

where $q_{\mathrm{lg}}$ is the interfacial heat transfer rate, $h_{\mathrm{lg}}$ is interfacial heat transfer coefficient and $A_{\mathrm{lg}}$ is the corresponding interfacial heat transfer area between gas and liquid phases.

The heat transfer coefficients on air and fluid side (natural convection) are a function of Raleigh, Prandtl and Grashof numbers. The heat transfer correlations used for vertical and horizontal cylindrical vessels are given below.

Vertical vessels:

$$
\begin{aligned}
& N u=0.59 \operatorname{Pr}^{0.25} G r^{0.25}, R a<10^{9} \\
& N u=0.129 \operatorname{Pr}^{0.33} G r^{0.33}, R a \geq 10^{9}
\end{aligned}
$$

Horizontal vessels:

$$
\begin{aligned}
& N u=0.525 \operatorname{Pr}^{0.25} G r^{0.25}, R a<10^{9} \\
& N u=0.129 \operatorname{Pr}^{0.33} G r^{0.33}, R a \geq 10^{9}
\end{aligned}
$$

In the above equations $\operatorname{Pr}=\mu \frac{C_{p}}{K}$ is the Prandtl number, $G r$ is the Grashof number given by $G r=\frac{g \beta \Delta T \rho^{2} l^{3}}{\mu^{2}}$ and $R a=\operatorname{Pr} G r$ is the Raleigh number. The heat transfer coefficient is then calculated as $h=N u \frac{K}{D}$ where $D$ is the characteristic length. For a horizontal cylinder, $D$ is the diameter and for the vertical cylinder the same becomes its length. Also $\beta$ is the thermal expansion coefficient of the fluid, $\mu$ is the viscosity and $\rho$ is the density.

The conduction heat transfer equation to be solved in the differential form is given by

$$
\frac{d T}{d t}=\alpha \frac{d^{2} T}{d x^{2}},
$$

where $\alpha=\frac{K}{\rho C_{p}}$ is the thermal diffusivity of the material. The above differential equation is discretized using central differencing scheme and solved using the boundary conditions based on heat transfer on the air and fluid sides on the vessel. 

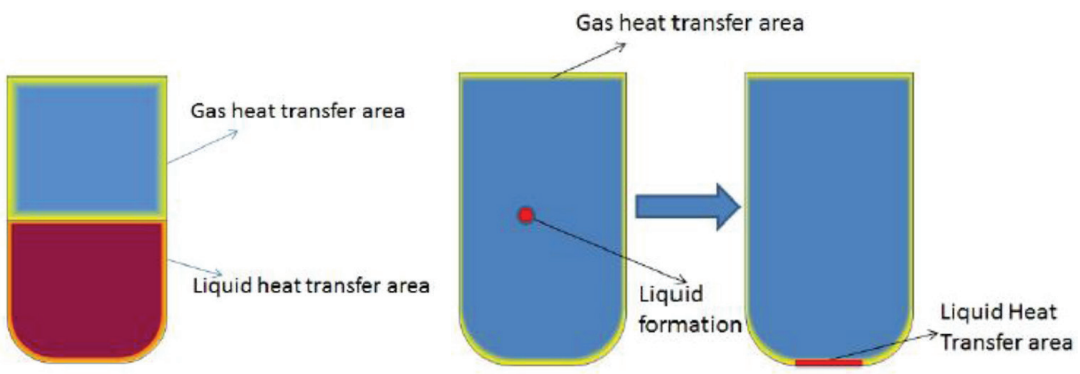

Figure 3: Heat transfer area used in calculations: (left) heat transfer area for bulk liquid and gas and (right) for very small liquid fractions, the thin film deposited at the bottom of tank leads to large heat transfer rates.

While calculating the heat transfer rates, a particular area of concern is the calculation of heat transfer area for liquid droplets. The heat transfer area for bulk liquid is the area of the container in contact with the liquid as shown in Fig. 3. In many simulations, the container may be filled with pressurized gas. As the pressure drops due to leak or blowdown, the temperature drops and the liquid may start to form. If the liquid volume is too small compared with the volume of the container, as an example when liquid is in the form of droplets, it is difficult to know the heat transfer area. Generally for liquid, it is assumed to settle down at the bottom of the container. With this assumption, for very small liquid quantity, it will be spread at the tank bottom as a very thin film. Since, the specific heat for liquid is larger than gas; suddenly a large amount of heat is transferred in to the container. This leads to spikes in the internal energy of the system that further influences the calculations. To overcome this problem a switch has been introduced in the model for initiating the heat transfer calculations.

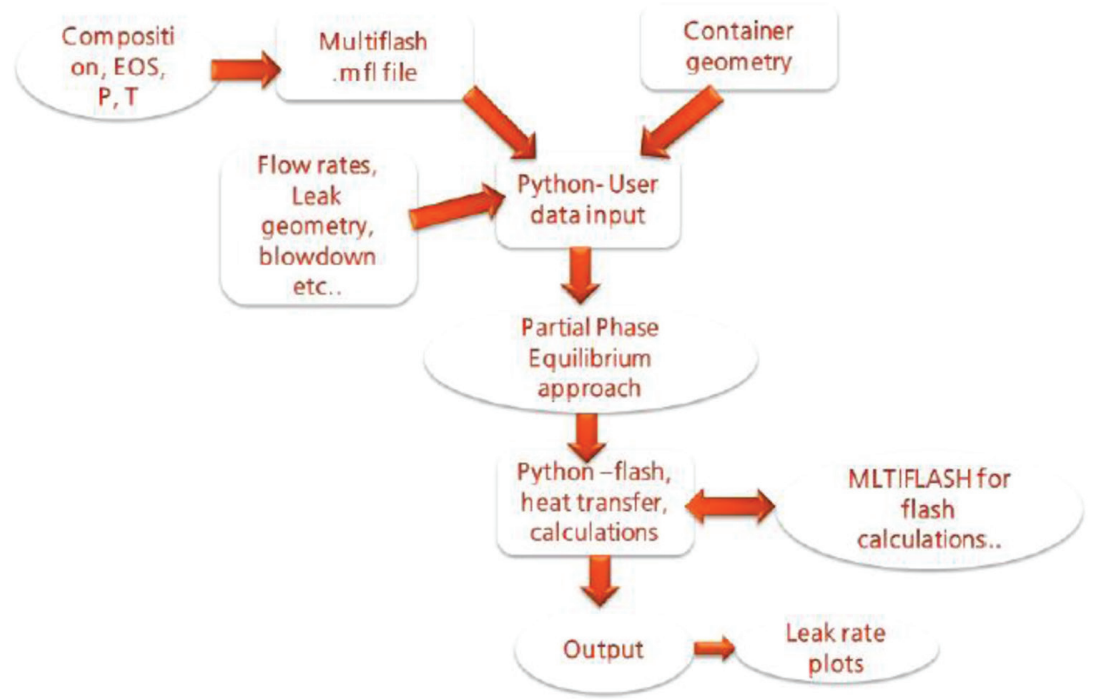

Figure 4: High level architecture of the mathematical model. 
The heat transfer to the liquid occurs only if the liquid is present for more than $1 \mathrm{~mm}$ height. Otherwise, the liquid is assumed to be suspended in gas as small droplets.

\subsection{Numerical implementation}

The above-mentioned sections describe the mathematical equations used in various calculations. The equations for energy, flow and temperature need to be coupled and solved at every time step. The model is developed in the Python programming language. A DLL is used to interact with the Multiflash software in order to carry out the VLE calculations. A high level structure of the numerical model is as shown in Fig. 4.

\section{VALIDATION}

The model predictions were validated against extensive available sets of experimental data in order to test the accuracy over a wide range of operating conditions. The test results were selected from various publications. The major publications referred for the blowdown experiments in hydrocarbon containers are DÁlessandro et al. [9], Speranza et al. [10] and Haque et al. [11]. Most of the experimental cases are already compiled in the publication presented by DÁlessandro et al. [9]. The abovementioned publications discuss the blowdown experiments. There was no experimental data found for pure liquid leaks.

\subsection{Experiment I1: blowdown of pure nitrogen gas}

The 'I1' experiment considered the blowdown of pure nitrogen from the top of a vertical vessel. Since the phase equilibrium calculations need multiple components, a small mole fraction of methane of $0.1 \%$ is added. The experiment was originally published by Haque et al. [11] and was referred to by DÁlessandro et al. [9]. The temperature of the tank is $290 \mathrm{~K}$ and pressure 190 bara. The ambient temperature is $290 \mathrm{~K}$. The diameter, height and wall thickness is $0.273,1.524$ and $2.5 \mathrm{~cm}$, respectively. The blowdown orifice diameter is 0.635 $\mathrm{cm}$. The backpressure in the blowdown system is $1 \mathrm{~atm}$.

The variations with the time of the pressure, bulk gas temperature and inside wall temperature are shown in Fig. 5. The vessel depressurized to atmospheric pressure in approximately

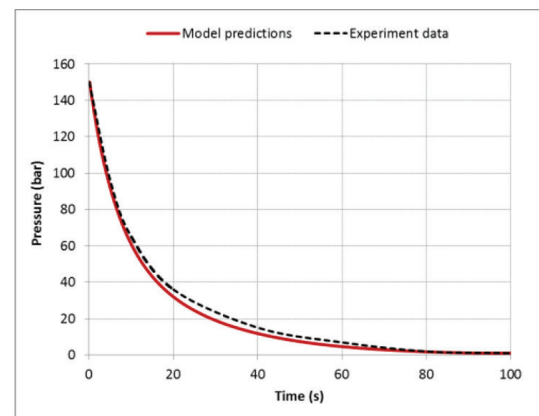

(a)

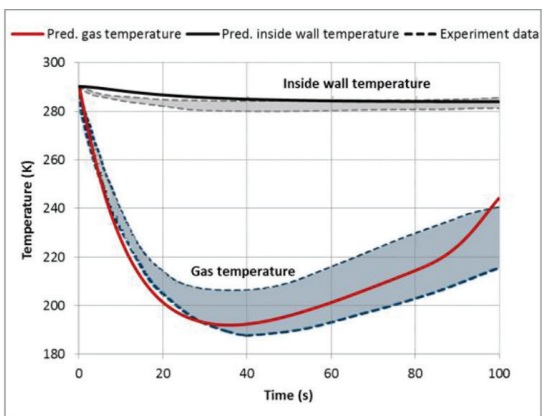

(b)

Figure 5: The variations of (a) the vessel pressure and (b) the bulk gas and inside wall temperature with time for the Experiment I1: blowdown of pure nitrogen. The dashed lines indicate experimental measurements, while the solid lines are the predictions. 
$100 \mathrm{~s}$, and the gas rapidly cooled during this expansion. Heat is transferred between the fluid and the wall; however, the wall temperature does not reduce nearly as much as the fluid does. The experimental results show a range of temperatures, as there are some spatial variations in the measured temperatures. The predicted pressure variation inside the tank, gas and inside wall temperatures compare well with the experimental data.

\subsection{Experiment S9: blowdown of a hydrocarbon mixture}

In the 'S9' experiment, a vertical vessel was blown down from the top. These experiments used mixtures of methane, ethane and propane (mole fractions $0.855,0.045$ and 0.1 , respectively). The composition contained trace amounts of higher molecular weight hydrocarbons, in particular butane, which were not reported in the literature [1]. The experiments were originally carried out by Haque et al. [11] and were later referred to by DÁlessandro [9]. The temperature of the tank is $303 \mathrm{~K}$ and pressure 120 bara. The diameter, height and wall thickness of the tank is $1.13 \mathrm{~m}, 3.24 \mathrm{~m}$ and $5.9 \mathrm{~cm}$, respectively. The blowdown orifice diameter is $1 \mathrm{~cm}$. The ambient temperature is $293 \mathrm{~K}$. The back pressure in the blowdown system is $1 \mathrm{~atm}$.

Figure 6 shows the experimental measurements and model predictions that include the time of the pressure in the vessel, the bulk gas and liquid temperatures during depressurization. The bulk liquid temperature was not measured in this experiment. Initially, the fluid in the vessel was only gas as shown in Fig. 7. As the blowdown proceeded, the pressure and temperature of the gas decreased. After approximately $100 \mathrm{~s}$, liquid condensate formed and the droplets fell to the bottom of the vessel to form a pool of liquid. Droplets continued to condense as the bulk gas temperature decreased during blowdown. As heat was transferred (it is easier to transfer heat to liquid than to gas from the vessel wall), the initial liquid temperature was higher than that of the gas. However, as the blowdown continued, the depressurization rate decreased and therefore the bulk gas temperature did not decrease rapidly. Eventually, the bulk gas temperature started to increase as heat was gained from the vessel wall more quickly than when it was lost due to expansion. Consequently, the bulk liquid temperature became lower than the bulk gas temperature; therefore, the vessel wall had a lower temperature when in contact with the liquid. There is a very good agreement between the predicted

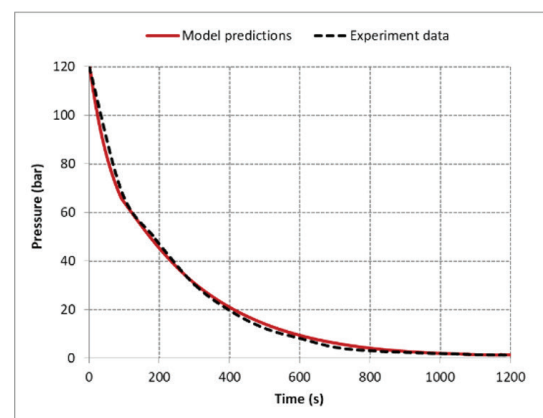

(a)

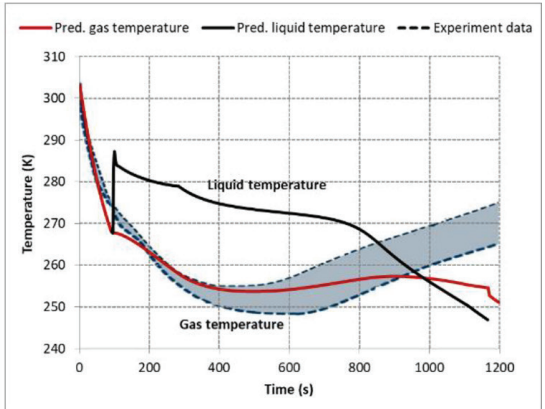

(b)

Figure 6: The variations of (a) the vessel pressure and (b) the bulk gas and liquid temperatures with time for the Experiment S09: blowdown of hydrocarbon mixture. The dashed lines indicate experimental measurements, while the solid lines are the predictions. 


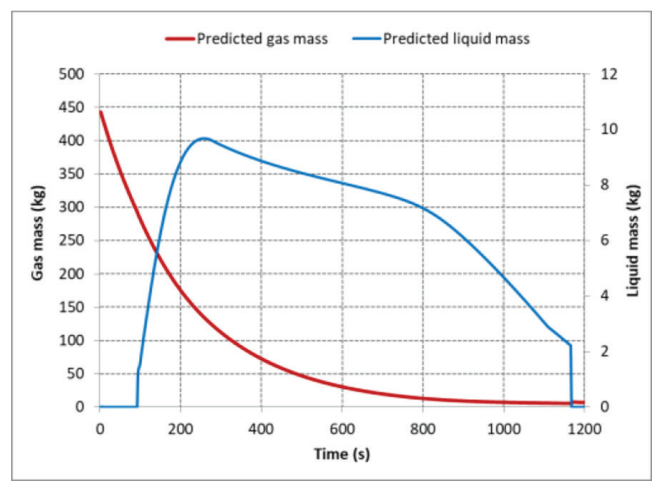

Figure 7: The predicted variations of the container gas and liquid mass with time for the Experiment S09: blowdown of hydrocarbon mixture.

results and experimental measurements of bulk gas temperature. However, some deviations are observed in predicted and measured bulk gas temperature in the latter part of the depressurization process.

\subsection{Experiment S12: blowdown of a hydrocarbon mixture}

In the 'S12' experiment, the same vertical vessel previously used in ' $\mathrm{S} 9$ ' experiment was blown down from the top with different initial composition. The experimental measurements were initially presented by Haque et al. [11] and later referred by DÁlessandro [9]. In this case, the initial hydrocarbon mixture contains methane, ethane and propane. The composition of initial hydrocarbon mixture is $66.5 \%$ methane, $3.5 \%$ ethane and $30 \%$ propane in mole percent. The initial temperature of the tank is $290 \mathrm{~K}$ and the initial pressure is 120 bara. The ambient temperature is $293 \mathrm{~K}$. The back pressure in the blowdown system is $1 \mathrm{~atm}$. The diameter, height and wall thickness of the tank is $1.13 \mathrm{~m}, 3.24 \mathrm{~m}$ and $5.9 \mathrm{~cm}$, respectively. The blowdown orifice diameter is $1 \mathrm{~cm}$.

Figure 8 shows the experimental measurements and model predictions that include the time of the pressure in the vessel, and the bulk gas and liquid temperatures for experiment 'S12'. Initially, the fluid in the vessel was only gas as shown in Fig. 9. Compared to the 'S9' experiment, significantly more liquid condensed from the gas in the 'S12' experiment. This leads to lower temperatures of the bulk liquid and gas phases inside the container. The temperature seems to be the most sensitive parameter in these calculations. It is observed that small variations in operating conditions or compositions lead to significant variations in temperature. As shown in Fig. 8(b), there was very little spatial variation in the bulk liquid temperature as there was for the bulk gas temperature. The original authors attributed this to the intense boiling in the liquid phase, which resulted in rapid mixing and thermal equilibrium in the phase. Therefore, it is essential to include this phenomenon in the model [1]. There is very good agreement between the predicted results and experimental measurements of bulk gas and liquid phase temperatures. However, some deviations are observed in predicted and measured bulk gas temperature in the latter part of the depressurization process. 


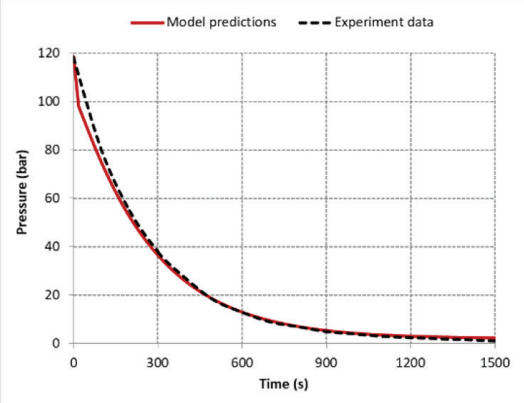

(a)

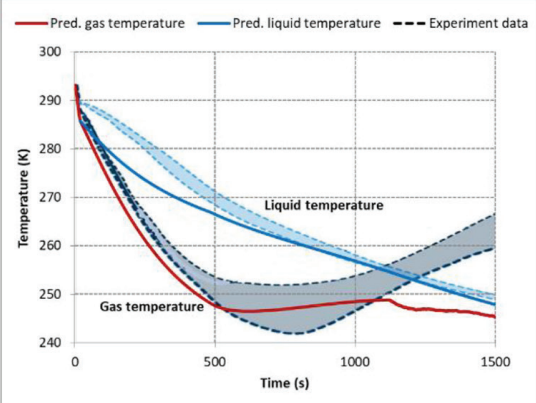

(b)

Figure 8: The variations of (a) the vessel pressure and (b) the bulk gas and liquid temperatures with time for the Experiment S12: blowdown of hydrocarbon mixture. The dashed lines indicate experimental measurements, while the solid lines are the predictions.

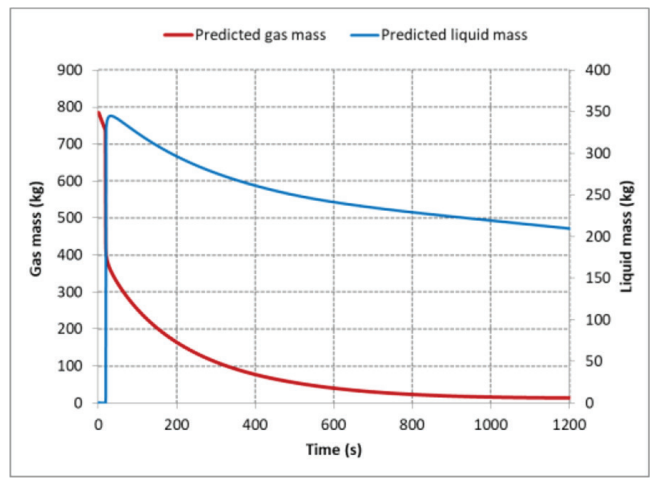

Figure 9: The predicted variations of the container gas and liquid mass with time for the Experiment S12: blowdown of hydrocarbon mixture.

\section{CONCLUSIONS}

The hydrocarbon leaks from process systems potentially lead to hazardous consequences with regard to human safety, environmental pollution and valuable assets. One common method for limiting the consequences associated with a process emergency is the rapid depressurization or blowdown of pressurized process systems. However, the blowdown process comes with its own challenges. There is experimental evidence that the assumption of thermodynamic equilibrium is not appropriate, since the two phases show an independent temperature evolution due to differences in heat transfer process. This paper presents a model for the simulation of the blowdown of pressurized vessels containing two-phase (gas-liquid) hydrocarbon fluids, considering non-equilibrium between phases. Two phases may be present either already at the beginning of the blowdown process (for instance, in gas-liquid separators) or as the liquid is formed from flashing of the vapour due to the cooling induced by pressure decrease. In addition, the transient behaviour of hydrocarbon leaks from pressurized process systems during depressurization is also included in the model providing the inputs for risk assessments. The model is based on a compositional approach, and it takes into 
account coupled effects of internal heat and mass transfer processes, as well as heat transfer with the vessel wall and the external environment. Numerical simulations show a generally good agreement with experimental measurements. The model is currently being tested on industrial cases to further investigate the importance of including thermodynamic behaviour in the leak calculations for quantitative risk analyses. Other improvements to the model that are under investigation are the addition of radiation effects and the inclusion of water in the thermodynamic model.

\section{REFERENCES}

[1] Fischer, B. \& Biswas, S., Validation of BLOWDOWN Technology in V9 of Aspen HYSYS $^{\circledR}$, Aspen HYSYS, White Paper.

[2] Vinnem, J.E., Offshore risk assessment. Principles, Modelling and Applications of QRA Studies, Springer-Science and Business Media, B.V., 1999.

https://doi.org/10.1007/978-94-017-2471-5

[3] Reynolds, W.C. \& Kays, W.M., Blowdown and charging processes in a single gas receiver with heat transfer. Trans ASME, 80, pp. 1160-68, 1958.

[4] Montgomery, G., How to predict temperatures during gas depressuring. Hydrocarbon Processing, pp. 85-88, 1995.

[5] Haque, M.A., Richardson, G., Chamberlain, S.M. \& Saville. G., Blowdown of pressurevessels. 2: Computer Model and Case Studies. Process Saf. Environ. Protect, 70(B), pp. 10-17, 1992.

[6] Overa, S.J., Stange, E. \& Salater, P., Determination of temperatures and flare rates during depressurization and fire. Proceedings of 72th GPA Annual Convention, pp. 235-47, 1994.

[7] Speranza, A. \& Terenzi, A., Blowdown of hydrocarbons pressure vessel with partial phase separation. Advances in Mathematics for Applied Sciences, 69, 2005. https://doi.org/10.1142/9789812701817_0046

[8] Wong, S.M.A., Development of a mathematical model for blowdown of vessels containing multicomponent hydrocarbon mixtures. PhD Thesis, University College London, 1998.

[9] DÁlessandro, V., Giacchetta, G., Leporini, M., Marchetti, B. \& Terenzi, A., Modelling blowdown of pressure vessels containing two-phase hydrocarbons mixtures with the partial phase equilibrium approach. Chemical Engineering Science, 126, 719-29, 2015. https://doi.org/10.1016/j.ces.2015.01.019

[10] Speranza, A. \& Terenzi, A., Blowdown of hydrocarbons pressure vessel with partial phase separation. Series on Advances in Mathematics for Applied Sciences, World Scientific, 69, 508, 2005. https://doi.org/10.1142/9789812701817_0046

[11] Haque, A., Richardson, S., Saville, G. \& Chamberlain, G., Rapid depressurization of pressure vessels. Journal of Loss Prevention in the Process Industries, 3(1), 4-7, 1990. https://doi.org/10.1016/0950-4230(90)85015-2

[12] Berge, G. \& Olstad, H., Experimental Study and Simulation on Heat Exposed Liquid Filled Process Equipment. 24th International Conference on Offshore Mechanics and Arctic Engineering, Halkidiki, Greece, 2005. 